East Asian Math. J.

Vol. 30 (2014), No.3, pp. 321-326

http://dx.doi.org/10.7858/eamj.2014.022

\title{
SOME REMARKS ON NON-SYMPLECTIC AUTOMORPHISMS OF K3 SURFACES OVER A FIELD OF ODD CHARACTERISTIC
}

\author{
JUNMYEONG JANG
}

\begin{abstract}
In this paper, we present a simple proof of Corollary 3.3 in [5] using the fact that for a K3 surface of finite height over a field of odd characteristic, the height is a multiple of the non-symplectic order. Also we prove for a non-symplectic CM K3 surface defined over a number field, the Frobenius invariant of the reduction over a finite field is determined by the congruence class of residue characteristic modulo the non-symplectic order of the K3 surface.
\end{abstract}

\section{Introduction}

Let $k$ be an algebraically closed field of odd characteristic $p$. Let $W$ be the ring of Witt vectors of $k$ and $K$ be the fraction field of $W$. Assume $X$ is a K3 surface over $k$. The second crystalline cohomology of $X, H_{c r i s}^{2}(X / W)$ is a free $W$-module of rank 22 equipped with a canonical Frobenius linear morphism

$$
\mathbf{F}: H_{c r i s}^{2}(X / W) \rightarrow H_{c r i s}^{2}(X / W) .
$$

Let $H_{\text {cris }}^{2}(X / K)=H_{\text {cris }}^{2}(X / W) \otimes K$ be the rational crystalline cohomology. If all the Newton slopes of F-isocrystal $\left(H_{\text {cris }}^{2}(X / K), F\right)$ are 1 , we say $X$ is supersingular. If $X$ is not supersingular, there exists an integer $h$ between 1 and 10 such that the slopes of $H_{c r i s}^{2}(X / K)$ are $1-1 / h, 1,1+1 / h$ of length $h, 22-h, h$ respectively. In this case, we say $X$ is of height $h$.

If $X$ is of finite height $h$, the Dieudonné module of the formal Brauer group of $X$ is

$$
\mathbb{D}\left(\widehat{B r}_{X}\right)=W[F, V] /\left(F V=p, F=V^{h-1}\right) .
$$

Here $\mathbb{D}\left(\widehat{B r}_{X}\right)$ is a free $W$-module of rank $h$ equipped with a Frobenius linear operator $F$ and a Frobenius-inverse linear operator $V$. The Dieudonné module

Received May 4, 2014; Accepted May 16, 2014.

2010 Mathematics Subject Classification. 11G25, 14J20.

Key words and phrases. K3 surface, Non-symplectic automorphism, Crystalline cohomology.

This research was supported by Basic Science Research Program through the National Research Foundation of Korea(NRF) funded by the Ministry of Education, Science and Technology(2011-0011428). 
$\mathbb{D}\left(\widehat{B r}_{X}\right)$ is isomorphic to $H^{2}\left(X, W \mathcal{O}_{X}\right)([1]) . H_{c r i s}^{2}(X / W)$ has an $F$-crystal decomposition according to the Newton slopes

$$
H_{c r i s}^{2}(X / W)=H_{c r i s}^{2}(X / W)_{[1-1 / h]} \oplus H_{c r i s}^{2}(X / W)_{[1]} \oplus H_{c r i s}^{2}(X / W)_{[1+1 / h]} .
$$

Here

$$
H_{c r i s}^{2}(X / W)_{[1-1 / h]}=\mathbb{D}\left(\widehat{B r}_{X}\right)
$$

and

$$
H_{c r i s}^{2}(X / W)_{[1+1 / h]}=\operatorname{Hom}\left(H^{2}\left(X, W \mathcal{O}_{X}\right), W\left(p^{2}\right)\right) .
$$

By the cup product pairing, $H_{c r i s}^{2}(X / W)_{[1-1 / h]}$ and $H_{c r i s}^{2}(X / W)_{[1+1 / h]}$ are isotropic and dual to each other. $H_{c r i s}^{2}(X / W)_{[1]}$ is unimodular. The discriminant of the $\mathbb{Z}_{p}$-lattice $H_{c r i s}^{2}(X / W)_{[1]}^{F=p}$ is $(-1)^{h+1}([10])$. The image of the cycle map

$$
N S(X) \otimes W \hookrightarrow H_{c r i s}^{2}(X / W)
$$

sits inside $H_{\text {cris }}^{2}(X / W)_{[1]}$. Therefore the Picard number of $X$, the rank of $N S(X)$ is at most $22-2 h$. Let $T_{\text {cris }}(X)$ be the orthogonal complement of the embedding $N S(X) \hookrightarrow H_{c r i s}^{2}(X / W)$. We call $T_{\text {cris }}(X)$ the crystalline transcendental lattice of $X$. By the above observation, we can see $H_{c r i s}^{2}(X / W)_{[1-1 / h]} \oplus$ $H_{c r i s}^{2}(X / W)_{[1+1 / h]}$ is a direct factor of $T_{c r i s}(X)$. From the exact sequence of sheaves on $X$

$$
0 \rightarrow W \mathcal{O}_{X} \stackrel{V}{\rightarrow} W \mathcal{O}_{X} \rightarrow \mathcal{O}_{X} \rightarrow 0,
$$

we have a canonical morphism

$$
H_{\text {cris }}^{2}(X / W)_{[1-1 / h]}=\mathbb{D}\left(\widehat{B r}_{X}\right) \simeq H^{2}\left(X, W \mathcal{O}_{X}\right) \rightarrow H^{2}\left(X, \mathcal{O}_{X}\right)
$$

Let

$$
\chi_{\text {cris }, X}: \text { Aut } X \rightarrow O\left(T_{\text {cris }}(X)\right)
$$

and

$$
\rho_{X}: \text { Aut } X \rightarrow G l\left(H^{0}\left(X, \Omega_{X / k}^{2}\right)\right)
$$

be the representation of Aut $X$ on the crystalline transcendental lattice and on global 2 forms. The images of $\chi_{c r i s, X}$ and $\rho_{X}$ are finite and there is a canonical projection $([5])$

$$
\operatorname{Im} \chi_{c r i s, X} \rightarrow \operatorname{Im} \rho_{X} .
$$

If $X$ is a supersingular K3 surface over $k$, the rank of $N S(X)$ is $22([2],[6])$. The cycle map $N S(X) \otimes W \hookrightarrow H_{c r i s}^{2}(X)$ is an embedding of $W$-lattices of same rank and we have

$$
N S(X) \otimes W \subset H_{\text {cris }}^{2}(X / W) \subset N S(X)^{*} \otimes W .
$$

The quotient $H_{c r i s}^{2}(X / W) /(N S(X) \otimes W)$ is a $\sigma$ dimensional $k$-space for an integer $\sigma$ between 1 and 10. We say $\sigma$ is the Artin-invariant of $X$. It is known that $N S(X)$ is completely determined by $p$ and $\sigma([12])$. We denote the NeronSeveri lattice of a supersingular K3 surface of Artin invariant $\sigma$ over a field of 
characteristic $p$ by $\Lambda_{p, \sigma}$. For a lattice $L$, let $d(L)$ be the discriminant of $L$. The discriminant $d\left(\Lambda_{p, \sigma}\right)$ is $-p^{2 \sigma}$. It is also known that there is a decomposition

$$
\Lambda_{p, \sigma} \otimes \mathbb{Z}_{p}=E_{0}(p) \oplus E_{1} .
$$

Here $E_{0}$ and $E_{1}$ are unimodular $\mathbb{Z}_{p}$-lattices of rank $2 \sigma$ and $22-2 \sigma$ respectively. And $d\left(E_{0}\right)=(-1)^{\sigma} \delta$ and $d\left(E_{1}\right)=(-1)^{\sigma+1} \delta$, where $\delta$ is a non-square unit of $\mathbb{Z}_{p}$. Note that a unimodular $\mathbb{Z}_{p}$-lattice is uniquely determined up to isomorphism by the rank and the discriminant, square or non-square.

We denote the characteristic polynomial over an indeterminate $T$ of a linear endomorphism $L$ by $\varphi(L)$. When $\alpha \in$ Aut $X$ is an automorphism of a K3 surface of $X, \varphi\left(\alpha^{*} \mid H_{c r i s}^{2}(X / W)\right)$ is a polynomial with integer coefficients ([4], 3.7.3). If $X$ is of finite height, $\varphi\left(\chi_{c r i s, X}\right)$ is also an integral polynomial. An automorphism $\alpha \in$ Aut $X$ is symplectic if $\rho_{X}(\alpha)=1$. An automorphism $\alpha \in$ Aut $X$ is purely non-symplectic if $\alpha$ is of finite order and the order of $\alpha$ is equal to the order of $\rho_{X}(\alpha)$. For $\alpha \in$ Aut $X$, we say the order of $\rho_{X}(\alpha)$ is the non-symplectic order of $\alpha$. Also, we call the order of $\operatorname{Im} \rho_{X}$ the non-symplectic order of $X$. An automorphism $\alpha \in \operatorname{Aut} X$ is tame if $\alpha$ is of finite order and the order of $\alpha$ is not divisible by $p$. An automorphism of finite order which is divisible by $p$ is called a wild automorphism. It is known that if $p>11$, there is no wild automorphism ([3]). When $X$ is of finite height, an automorphism $\alpha \in$ Aut $X$ is weakly tame if the order of $\chi_{c r i s, X}(\alpha)$ is not divisible by $p$. Every tame automorphism is weakly tame. Since $\chi_{c r i s, X}(\alpha)$ is of finite order, all roots of $\varphi\left(\chi_{c r i s, X}(\alpha)\right)$ are roots of unity. Hence if a primitive $n$-th root of unity is an eigenvalue of $\chi_{c r i s, X}(\alpha)$, the rank of $T_{c r i s}(X)$ is at least $\phi(n)$ because $\varphi\left(\chi_{\text {cris }, X}(\alpha)\right) \in \mathbb{Z}[T]$. Therefore when $X$ is of finite height and $p \geq 23$, every automorphism of $X$ is weakly tame.

If $X$ is of height $h, \alpha$ is a weakly tame automorphism of $X$ and $\rho(\alpha)=\xi$ is of order $n$, then all the eigenvalues of $\alpha^{*} \mid H_{\text {cris }}^{2}(X / W)_{[1-1 / h]} \oplus H_{\text {cris }}^{2}(X / W)_{[1+1 / h]}$ are $\xi^{ \pm 1}, \xi^{ \pm p^{-1}}, \cdots, \xi^{ \pm p^{1-h}}$ up to multiplicity ([5], Theorem 2.9). By this and an argument based on the Tate conjecture for K3 surfaces ([9], [6]), we also proved the following.

Proposition([5], Corollary 3.3) Let $k$ be an algebraically closed filed of odd characteristic $p$. Let $X$ be a K3 surface over k equipped with an automorphism $\alpha \in \operatorname{Aut}(X)$ such that the order of $\rho_{X}(\alpha)$ is $N>2$. We assume the rank of the Neron-Severi group of $\mathrm{X}$ is at least $22-\phi(N)$. If $p^{m} \equiv-1$ modulo $N$ for some $m, X$ is supersingular. If $p^{m} \not \equiv-1$ modulo $N$ for any $m$ and the order of $\mathrm{p}$ in $(\mathbb{Z} / N \mathbb{Z})^{*}$ is $n, X$ is of height $n$.

In the next section, we present a simple proof of the above proposition. For that, we prove the following theorem. 
Theorem 2.1. Let $k$ be an algebraically closed field of odd characteristic $p$. Assume $X$ is a K3 surface over $k$ and $N>2$ is the non-symplectic order of $X$. If $p^{m} \not \equiv-1$ modulo $N$ for any $m, X$ is of finite height. In this case, if $n$ is the order of $p$ in $(\mathbb{Z} / N)^{*}$, the height of $X$ is a multiple of $n$.

When $X$ is a complex algebraic K3 surface, the transcendental lattice of $X$ is the orthogonal complement of the embedding

$$
N S(X) \hookrightarrow H^{2}(X, \mathbb{Z}) .
$$

We denote the transcendental lattice of $X$ by $T(X)$. When $\rho(X)$ is the Picard number of $X$, the signature of $T(X)$ is $(2,20-\rho(X))$. If $N$ is the non-symplectic order of $X$, the rank of $T(X)$ is a multiple of $\phi(N)([7])$. We say $X$ is a nonsymplectic CM K3 surface of order $N$ if $\operatorname{rank} T(X)$ is equal to $\phi(N)$. A nonsymplectic CM K3 surface gives a CM point in a moduli Shimura variety, so it has a model over a number field ([11]). It seems like that there are only few non-symplectic CM K3 surfaces. Also it is known that there is a unique nonsymplectic CM K3 surface of order $N$ for many $N$. In a previous work ([5]), if $X$ is a non-symplectic CM K3 surface of order $N$ with $\phi(N)>10$ and $\operatorname{Im} \rho_{X}$ is generated by a purely non-symplectic automorphism, we proved that the height and the Artin invariant of a reduction of $X$ over a field of odd characteristic $p$ is determined by the congruence class of $p$ modulo $N$. In the next section, we give a generalization of this result for an arbitrary non-symplectic CM K3 surface.

\section{Results}

Theorem 2.1. Let $k$ be an algebraically closed field of odd characteristic $p$. Assume $X$ is a K3 surface over $k$ and $N>2$ is the non-symplectic order of $X$. If $p^{m} \not \equiv-1$ modulo $N$ for any $m, X$ is of finite height. In this case, if $n$ is the order of $p$ in $(\mathbb{Z} / N)^{*}$, the height of $X$ is a multiple of $n$.

Proof. If $X$ is a supersingular K3 surface of Artin-invariant $\sigma$, the non-symplectic order of $X$ divides $p^{\sigma}+1$ ([8], Theorem 2.1). Hence under the assumption, $X$ is not supersingular. Let us choose an automorphism $\alpha \in$ Aut $X$ such that $\operatorname{Im} \rho_{X}$ is generated by $\rho_{X}(\alpha)$. If the order of $\chi_{c r i s, X}(\alpha)$ is $p^{r} M$ for $r, M \in \mathbb{N}$ with $p \nmid M, \alpha^{p^{r}}$ also generates $\operatorname{Im} \rho_{X}$ and is weakly tame. After replacing $\alpha$ by $\alpha^{p^{r}}$, we may assume $\alpha$ is weakly tame. Suppose the height of $X$ is $h$. If $\xi=\rho_{X}(\alpha)$, by $\left([5]\right.$, Theorem 2.9), all the eigenvalues of $\alpha^{*} \mid H_{c r i s}^{2}(X / W)_{[1-1 / h]}$ are $\xi^{-1}, \xi^{-1 / p}, \cdots, \xi^{-1 / p^{h-1}}$ up to multiplicity. On the other hand, if $\alpha^{*} x=\lambda x$ for some $x \in H_{\text {cris }}^{2}(X / W)_{[1-1 / h]}$,

$$
\alpha^{*}(V x)=V\left(\alpha^{*} x\right)=V(\lambda x)=\lambda^{1 / p} V x .
$$

Therefore for any $i \in \mathbb{Z}, \xi^{-1 / p^{i}}$ is an eigenvalue of $\alpha^{*} \mid H_{c r i s}^{2}(X / W)_{[1-1 / h]}$ and the rank of eigenspace for the eigenvalue $\xi^{-1 / p^{i}}$ is equal to the rank of 
eigenspace for the eigenvalue $\xi^{-1}$. Hence the height $h$ is divided by the order of $p$ in $(\mathbb{Z} / N)^{*}$.

Corollary 2.2. Let $k$ be an algebraically closed filed of odd characteristic $p . X$ is a $\mathrm{K} 3$ surface over $\mathrm{k}$ equipped with an automorphism $\alpha \in \operatorname{Aut}(X)$ such that the order of $\rho_{X}(\alpha)$ is $N>2$. We assume the rank of the Neron-Severi group of $\mathrm{X}$ is at least $22-\phi(N)$. If $p^{m} \not \equiv-1$ modulo $N$ for any $m$ and the order of $p$ in $(Z / N Z)^{*}$ is $n, X$ is of height $n$.

Proof. By Theorem 2.1, $X$ is of finite height and the height of $X$ is a multiple of $n$. By the assumption, the rank of $T_{\text {cris }}(X)$ is $\phi(N)$. Since $\varphi\left(\chi_{\text {cris }, X}(\alpha)\right)$ is an integral polynomial of degree $\phi(N)$ and a primitive $N$-th root of unity is an eigenvalue of $\chi_{c r i s, X}(\alpha), \varphi\left(\chi_{c r i s, X}(\alpha)\right)$ is the $N$-th cyclotomic polynomial. It follows that every eigenvalue of $\chi_{c r i s, X}(\alpha)$ occurs only one time. Considering ([5], Theorem 2.9), the height of $X$ is at most $n$. Therefore the height of $X$ is $n$.

Let $X$ be a non-symplectic CM K3 surface of order $N$. We may assume $X$ is defined over a number field $F$. Let us fix a smooth projective integral model $X_{R}$ of $X$ over an integer ring $R$ with $\operatorname{Spec} R \subset \operatorname{Spec} \mathfrak{o}_{F}$. For each place $v \in \operatorname{Spec} R$, let $p_{v}$ be the residue characteristic of $v$. We assume $p_{v} \nmid N$ and $p_{v}$ is unramified in $F$ for any $v \in \operatorname{Spec} R$. We denote the reduction of $X_{R}$ over the residue field $k(v)$ by $X_{v}$.

Theorem 2.3. If $p_{v}^{m} \not \equiv-1$ modulo $N$ for any $m \in \mathbb{Z}, X_{v}$ is of finite height and the height of $X_{v}$ is the order of $p_{v}$ in $(\mathbb{Z} / N)^{*}$. If $p_{v}^{m} \equiv-1$ for some $m \in \mathbb{Z}, X_{v}$ is supersingular. Moreover if $p_{v}$ does not divide $d(N S(X))$, the Artin-invariant of $X_{v}$ is the half of the order of $p_{v}$ in $(\mathbb{Z} / N)^{*}$.

Proof. There is an embedding

$$
N S(X) \hookrightarrow N S\left(X_{v}\right)
$$

so the rank of $N S\left(X_{v}\right)$ is at least $22-\phi(N)$. Let $N_{v}$ be the non-symplectic order of $X_{v}$. Then $N_{v}$ is a multiple of $N$. If $p_{v}^{m} \not \equiv-1$ modulo $N$ for any $m \in \mathbb{Z}, p_{v}^{m} \not \equiv-1$ modulo $N_{v}$, so $X_{v}$ is of finite height. Since the rank of $T\left(X_{v}\right.$ is at least $\phi\left(N_{v}\right), \phi(N)=\phi\left(N_{v}\right)$ and $N_{v}=N$ or $N_{v}=2 N$. In any case, the order of $p$ in $\left(\mathbb{Z} / N_{v}\right)^{*}$ is equal to the order of $p$ in $(\mathbb{Z} / N)^{*}$. By Corollary 2.2, the height of $X_{v}$ is the order of $p_{v}$ in $(\mathbb{Z} / N)^{*}$. Assume the order of $\xi=\rho_{X}(\alpha)$ is $N$ for some $\alpha \in \operatorname{Aut} X$. Let $T_{\text {cris }}(X)$ be the orthogonal complement of the embedding

$$
N S(X) \otimes W \hookrightarrow N S\left(X_{v}\right) \otimes W \hookrightarrow H_{\text {cris }}^{2}\left(X_{\text {upsilon }} / W\right) .
$$

Since $H_{\text {cris }}^{2}(X / W)$ is canonically isomorphic to $H_{d r}^{2}\left(X_{R} / R\right) \otimes W, \alpha^{*} \mid T_{\text {cris }}(X)$ is of finite order. If $X_{v}$ is of finite height, $T_{\text {cris }}\left(X_{v}\right) \subset T_{\text {cris }}(X)$ and all the eigenvalues of $\alpha^{*} \mid T_{\text {cris }}\left(X_{v}\right)$ are distinct. If $p^{m} \equiv-1$ modulo $N, \xi^{-1 / p^{m}}=\xi$ occurs as an eigenvalue of $\alpha^{*} \mid H_{\text {cris }}^{2}(X / W)_{[1-1 / h]}$. But $\xi$ is also an eigenvalue of $\alpha^{*} \mid H_{c r i s}^{2}(X / W)_{[1+1 / h]}$ and it is a contradiction. Therefore $X_{v}$ is supersingular. 
If $p$ does not divide $d(N S(X)), N S(X) \otimes W$ is unimodular and the Artininvariant of $X_{v}$ is at most $\phi(N) / 2$. Also when $\sigma$ is the Artin-invariant of $X_{v}, N$ divides $p^{\sigma}+1$, so $p^{\sigma} \equiv-1$ modulo $N$. We have an isomorphism $N S\left(X_{v}\right)^{*} / N S\left(X_{v}\right)=T_{\text {cris }}(X)^{*} / T_{\text {cris }}(X)$ compatible with the action of Aut $X$. By ([5], theorem 2.9), if $\sigma$ is greater than the half of the order of $p_{v}$ in $(\mathbb{Z} / N)^{*}$, $\xi$ appears more than twice in the eigenvalues of $\alpha^{*} \mid T_{\text {cris }}(X)$. Therefore the Artin-invariant of $X_{v}$ is the half of the order of $p_{v}$ in $(\mathbb{Z} / N)^{*}$.

Remark 2.4. If a non-symplectic CM K3 surface of order $N, X$ has a reduction of height $\phi(N) / 2$ or of Artin invariant $\phi(N) / 2$, the Legendre symbol $\left(\frac{d(N S(X))}{p}\right)$ is constant for all primes $p$ in a congruence class modulo $N$.

\section{References}

[1] Artin, M. and Mazur,B. Formal groups arising from algebraic varieties, Ann. Sci. École Norm. Sup(4) 10,1977,87-131.

[2] Francois, C. The Tate conjecture for K3 surfaces over finite fields, Invent. Math. 194, 2013, 119-145.

[3] Dolgachev, I. and Keum, J. Finite group of symplectic automorphisms of K3 surfaces in positive characteristic, Ann. of Math. 169, 2009, 269-313.

[4] Illusie, L. Report on crystalline cohomology, Algebraic Geometry, Proc. Sympos. Pure Math 29, 1975, 459-478.

[5] Jang, J. The representation of the automorphism groups on the transcendental cycles and the Frobenius invariants of K3 surface, arxiv:1312.7634.

[6] Madapusi Pera, K. The Tate conjecture for K3 surfaces in odd characterisitc, arXiv:1301.6326.

[7] Nukulin, V.V. Finite gropus of automorphisms of Kählerian K3 surfaces, Trudy Moskov. Mat. Obshch 38, 1979, 75-137.

[8] Nygaard, N.O. Higher De Rham-Witt complexes of supersingular K3 surfaces, Compositio Math. 42, 1981, 245-271.

[9] Nygaard, N.O. and Ogus, A. Tate conjecture for K3 surfaces of finite height, Ann. of Math.(2) 122, 1985, 461-507.

[10] Ogus, A. A crystalline Torelli theorem for supersingular K3 surfaces, Prog. Math. 36, 1983, 361-394.

[11] Rizov, J. Kuga-Satake abelian varieties of K3 surfaces in mixed characteristic, J.reine angew. Math. 648, 2010, 13-67.

[12] Rudakov, A.N. and Shafarevich,I.R. Surfaces of type K3 over fields of finite characteristic, Current problems in mathematics, Akad. Nauk SSSR, Vsesoyuz. Inst. Nauchn. i Tekhn. Informatsii vol. 18, 1981, 115-207.

JUNMYEONG JANG

Department of Mathematics University of Ulsan Daehakro 93, Namgu Ulsan 680-749, Korea

E-mail address: jmjang@ulsan.ac.kr 